\title{
Intragenomic polymorphism of internal transcribed spacer ITS1 in the locus 35S rRNA of polyploid Avena species
}

\author{
Rodionov A.V. ${ }^{1,2 *}$, Krainova L. ${ }^{2}$, Gnutikov A.A. ${ }^{1,3}$, Mikhailova Y. ${ }^{1}$, Machs E.M. ${ }^{1}$, \\ Shneyer V.S. ${ }^{1}$, Loskutov I.G. ${ }^{3}$, Muravenko O.V. ${ }^{4}$ \\ ${ }^{1}$ Komarov Botanical Institute, RAS, St. Petersburg, Russia \\ ${ }^{2}$ Department of Cytology and Histology, St. Petersburg State University, St. Petersburg, Russia \\ ${ }^{3}$ N.I. Vavilov All-Russian Institute of Plant Genetic Resources (VIR), St. Petersburg, Russia \\ ${ }^{4}$ Engelhardt Institute of Molecular Biology, RAS, Moscow, Russia \\ *e-mail:avrodionov@mail.ru
}

Using sequence-tagged Roche 454 platform, we studied intragenomic polymorphism of ITS1, a spacer region of the multiple 35SrRNA genes in four polyploid Avena species. Comparatison of ITS1 sequences from the diploid species, earlier sequenced by Sanger approach, allowed to detect two indels specific for all ITS1 of C-genome diploid species. It enabled easily identifying rare C-subgenome-specific ITSs among hundreds of ITS1 reads characteristic for the A-subgenomes. Instead of expected $50 \% \mathrm{C}$-variant reads of $35 \mathrm{~S}$ rDNA in A. insularis (karyotype AACC or CCDD) and $33 \% \mathrm{C}$-variant reads in hexaploids A. fatua, A. ludoviciana and A. sterilis (all AACCDD), the actual rate consisted only about $4 \%$ in $A$. insularis and $2-3 \% \mathrm{C}$-variant reads in hexaploid genomes. The C-genome-originated $18 \mathrm{~S}$ (fragment), ITS1 and 5.8S (small fragment) were 10 times more variable than the same sequences originated from to A-genome. Some of the sequences of $\mathrm{C}$-subgenomes contained deletions, including deletions in the 18S rRNA region. As for the origin of C-subgenome ITS1s in the polyploid species, some sequences revealed similarity to the ITS1 variants of either A. macrostachya $(\mathrm{Cm})$ or A. ventricosa $(\mathrm{Cv})$, but the core variant of $\mathrm{C}$-subgenome ITS1 on the genetic tree is approximately equidistant from all the present-day $\mathrm{C}$-genome diploid species. The A-type ITS1 of $A$. insularis is represented by few families, one of which is close to the A-genomes of A. longiglumis, A. hirtula and A. wiestii. As for hexaploids, there are two more frequent families of A-type rDNA reads in their genomes. We believe that one of them, represented by a higher number of reads, is located in NORs of D- and another one in NORs of A-subgenomes.

Acknowledgements: This study was supported by the RFBR KOMFI 17-00-00340 (17-00-00336, 17-00-00337, 17-00-00338). 